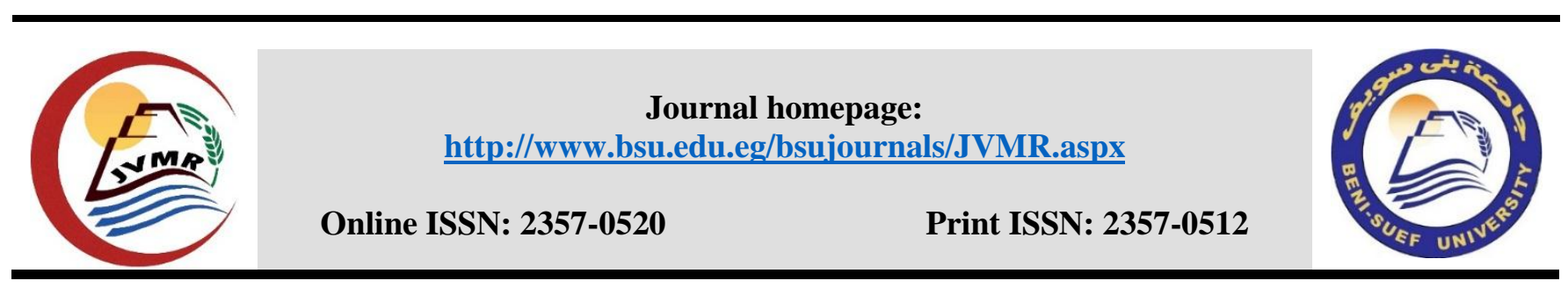

Original Research Article

\title{
Development of An Interactive Three-Dimensional Virtual Skeleton of Birds for Use in Anatomical Education
}

\author{
HebatAllah H. Mahmoud*; M.R. Gad; M.G. Tawfiek and A.S. Awaad \\ Department of Anatomy and Embryology, Faculty of Veterinary Medicine, Beni-Suef University, Beni-Suef \\ 62511, Egypt.
}

\section{ABSTRACT}

The aim of the present work was to describe the way of creating chosen interactive and animated three-dimensional anatomic virtual models of particular parts of birds' skeleton. These models can be used for teaching purposes in anatomy courses to assist veterinary students, and veterinarians to know the different structures of birds' bones without dissection. We used available multimedia technologies to develop a simple computer program for visualization and annotation of threedimensional duck skeleton. The entire program involves three-dimensional realistic objects, description texts, and images, in addition to quizzes for users' selfassessment. Addressing the anatomical structures made the program more useful to promote knowledge, enhance and facilitate teaching and learning of birds' osteology for veterinary students.

\footnotetext{
*Corresponding author. Department of Anatomy and Embryology, Faculty of

Veterinary Medicine, Beni-Suef University, Beni-Suef 62511, Egypt. E.mail:

d.hebatallah@yahoo.com
}

ARTICLE INFO

Article history:

Received 5/2018

Accepted 6/2018

Online 6/2018

Keywords:

Anatomy

Birds

Computer-assistedteaching

Skeleton

Three-dimensional visualization

\section{Introduction}

Anatomy is a discipline where spatial visualiza-tion is of importance. While anatomy textbooks and atlases provide static anatomical illustrat-ions in two dimensions, they are of limited value in exposing three-dimensional dynamics of anatomical structures. Hence, learning anatomy is essentially a three-dimensional endeavor, learners may find it difficult to visualize static images as three dimensions and understand certain dynamic aspects of anatomy.
In anatomy, students have to manipulate and rotate structures from different views to clearly identify anatomical structures Azer and Azer (2016).

By using virtual reality the student is able to inspect and interact directly with the anatomical structures, this experience has been really appreciated by both teachers and students Fairén et al., (2017).

In anatomy laboratories, cadaver dissection remains a straight forward and powerful approach to demonstrate and learn the three-dimensional 
physical structures and relevant relationships in a number of body regions and organs Azkue (2013). In the last few years, the use of animals' cadavers in anatomy classes has been reduced; hence alternative interactive teaching methods appeared using computer-based programs which are one of the successful alternatives. Use of computer programs compared to traditional anatomy teaching methods is recommended to enhance and facilitate learning ElNady et al., (2014); Codd and Choudhury (2011); Turney (2007); Galland et al., (1995).

The aim of this article is to describe the way of creating chosen interactive and animated threedimensional anatomic virtual models of particular parts of birds' skeleton. These models can be used for teaching purposes in anatomy courses to assist veterinary students, and veterinarians to know the different structures of birds' bones without dissection.

\section{Materials and methods}

Three-dimensional digital specimens were created using individual bones of an adult female Baladi duck. The steps in the current work were done according to Malinowski (2003) and Tawfiek (2011).

\subsection{Preparation of duck skeleton}

The individual bones of duck skeleton were prepared for maceration according to Hildebrand (1968).

The cadaver was skinned and the gut was removed. The larger muscle masses were removed, and the carcass was dismembered into pieces of convenient size taking care not to scar the bones. The flesh was removed from between the ribs. Wire was used to dislodge the spinal cord from the spinal canal. The wings and limbs were severed from the body but left each intact. The brain and two eyes were removed from the skull carefully.

For complete bacterial maceration: suitable containers were used and specimens tagged with string, covered well with water and left at warmer room for two weeks. The liquid in the containers was agitated and poured off. The bones were rinsed and covered with fresh water and allowed to sit again, this process was repeated until the bones were clean. Then the bones were cleaned using a hard brush to remove remnants of flesh and ligaments attached to the bones. The brushed bones were placed in 3\% hydrogen peroxide solution for five days, and then rinsed with water. Finally, the bones were allowed to dry at room temperature.

\subsection{Material collection}

A written resource contained the names of specimens and landmarks that a veterinary technology student must learn about the anatomy of birds' skeleton. This list was used as a guide during production. It was important that all of the mentioned landmarks be included in the final version of the project.

For each specimen in the project, it was necessary to thoroughly review all of the landmarks in a current anatomical atlas. The three publications used were: Anatomy of domestic birds, Nickel et al., (1977), color atlas of avian anatomy, Mclelland (1991) and Avian Anatomy, textbook and color atlas, Konig et al., (2016). This ensured the highest level of accuracy and avoided passing misinformation to the user.

\subsection{Scope}

The original goal of the project was to include models of duck skeleton in the production. These are the most important bones for an individual working in the veterinary profession to know. Each bone has many unique physical areas of importance, or anatomical landmarks. This list can be overwhelming for the student.

\subsection{Interface design}

A graphical user interface was created to integrate all the materials. It was designed to have an attractive appealing that is logical and easy for the student to use immediately, with little instruction and without any need of training.

\subsection{Treatment}

This educational project was intended to make learning duck skeleton anatomy fun and easy. The CD-ROM will give the students twenty-four hour access to high quality three dimension virtual specimens that can be manipulated and viewed from multiple angles, with specific landmarks able to be turned on or off at any time. The specimens can be rotated using the mouse much as they can be in real life using one's hands. The project can be used at any computer that is equipped with a CD-ROM drive or even using memory cards. Since the project is self-contained on a compact disc, it can be played anywhere independent of the availability of an internet connection or software plug-ins. 


\subsection{Software}

The main software programs used to create the project are: VR Worx 2.6, Adobe Photoshop CS6, Macromedia flash 8.0 and Autoplay Media Studio 8.0.1.1. The project was designed to run from $\mathrm{CD}$ ROM or memory cards on Microsoft windows platform.

\subsection{Hardware}

A single row Kaidan Magellan desktop turntable (Kaidan inc., 2001) with a pedestal on which the samples can be mounted is an integral component of the system. The object being photographed must be tightly secured to the pedestal and rotated to achieve the three-dimensional illusion.

A digital camera (Nikon-Coolpix 12.1-mega pixelblack, Model: P90, image resolution up to $4016 \mathrm{x}$ 3006 pixels, optical zoom 24x, digital zoom 4x) was fixed on a 3-way pan-head floor standing tripod. A diffuse fluorescent light source was used for illumination. A large piece of black felt material was placed behind the turntable to produce a black background to the specimens.

\subsection{Production}

This included photography techniques, three dimensional model creation and prototype usability testing. A total of fifteen specimens were processed and included in the final version of the project. This included a complete articulated skeleton, the main regions of the head (skull and mandible), bones of the wing (scapula, clavicle, coracoid, humerus, radius, ulna, carpus, metacarpus and phalanges), and bones of the pelvic limb (pelvic bone, femur, tibiotarsus, fibula, tarsometatarsus, toes).

Each specimen possesses a different number of anatomical landmarks that were individually researched and highlighted (Table 1).

\subsubsection{Photography process}

The digital camera was fixed on a tripod, allowing the images to be acquired without altering the camera's position. The camera was positioned approximately thirty centimeters from the specimens mounted on the turntable. The interval rate (10 degrees/shot) was manually adjusted with the motion of the turntable and after complete rotation of the turntable; thirty-six exposures of the camera took approximately ten minutes. The images were transferred directly from the camera to the computer, bypassing the need for a memory card reader. Each file generated had a resolution of $4000 \times 3000$ pixels and was approximately $5 \mathrm{MB}$ in size.

\subsubsection{Image editing}

Each image was imported into Adobe Photoshop for editing. A high tolerance is specified for the magic wand tool that was used to select and modify the background. The fill tool then used to create a uniform black background. The airbrush tool was used to touch up any areas not intended to be visible, such as the support pedestal. After each image was processed, its size was reduced to approximately 2.0 MB. This image dimension gives the best balance between specimen visibility and file size. The process was repeated for the remaining 35 images of each specimen. Edited images with a uniform black background and labels were imported into VR. Worx 2.6 software which organized the images up next to each other and stitched the images together. The result was a QuickTime movie that allowed the user to view a single row object from different angles.

\subsubsection{Assembly in Macromedia Flash}

The thirty-six jpg images composing a specimen were imported into the Macromedia Flash timeline. A ready to use actionscript progra mming was used to monitor the user's mouse movements to establish the user interactivity with the digital specimen.

The work on this project took approximately eight months to prepare the duck skeleton, capture photos, edit the images, stitch and integrate all images into the final project.

\section{Results}

A simple computer application was impleme nted here for visualization and annotation of threedimensional duck skeleton. The user will find an autoplay application with the label "3D_Duck_Skeleton." An action is needed from the user to press the left button of the mouse to run the project. The entire project consisted of an introduction page, main page, program and quizzes.

\subsection{Introduction}

When the program is launched, an introduction screen (Fig. 1) appeared showing the title of the entire work, and the user can install the required software (Macromedia flash, QuickTime and Shockwave players) for proper use of the contents on this compact disc. Also in the middle bottom of 
the screen, the user can get help on how to use this $\mathrm{CD}$ and have an idea about the references used during the preparation of the software program. To continue through the entire contents of the program, the user must click on a multistate button labeled "Main Menu" found on the right bottom of the screen.

\subsection{Main Menu}

On navigation of the main menu screen (Fig. 2), the user can interact directly with a flash movie of 2D image of the duck skeleton through moving the mouse over the different parts of the skeleton and the touched part will be highlighted with some information appeared beside the bird. On the left side of the screen, there is a list of different parts of duck skeleton demonstrating the bones which are incorporated in this $3 \mathrm{D}$ project and other parts which couldn't be made in a separate 3D movie; these should appeared on the whole skeleton movie. Also from this screen there are three buttons below the flash movie through which the user can return back to the introduction page, start to view the 3D objects or take the quiz after completion of the entire contents of this CD.

\subsection{Program}

By clicking on the "Start" button, the user will navigate 3D objects screen (Fig. 3), showing the 3D object in the middle of the screen, below which there are eight control buttons through which the user can move the object to the left side, right side, show labels, hide labels, zoom in object, zoom out object, return to the first position or auto rotate the object movie.

On the left of all screens in the program there are multistate buttons labeled "Duck skeleton, Skull, Thoracic limb and Pelvic limb" through which the user is free to move through different parts of the entire contents of this program.

On the lower left side of the screen, there is a multistate button labeled "Main Menu" through which the user can return to the navigation menu at any time he wants.

Below each 3D object movie, always there is a scrollable paragraph object of a written text giving the user data about the displayed 3D object. To navigate a high-quality QuickTime movie, the user can click on a button on the right bottom of the screen.

On pressing the button labeled "Thoracic limb" on the left side of the screen, another screen (Fig. 4) will appear showing eight buttons above the 3D object labeled "Scapula, Clavicle, Coracoid, Humerus, Radius, Ulna, carpo-metacarpus and digits". On pressing the button labeled "Pelvic limb" this will navigate to another screen (Fig. 5) with five buttons above the 3D object movie labeled "Pelvic bone, Femur, Tibiotarsus, Tarsometatarsal and digits (Toes)." Throughout the entire work, the user is free to finish the course at once, or he could minimize or even close the program through two buttons on the top right of all screens.

For ease and more convenience to the user, a menu bar with dropdown menus on the top of the screen contain all parts of the entire work, through which the user can click any part to navigate.

\subsection{Quiz}

After completion of this course, the user can answer the quiz on the main menu screen, or the menu bar on the top of the screen. This quiz (Fig. 6) allows the user to test him to what extent he understands and remembers the contents of the program. When the user presses the button labeled "Quiz," a screen will open showing different formats of these quizzes including; a true/false question which is a statement that is either true or false, followed by two choices, only one of which is correct. The quiz taker should choose the correct answer to get the points. A multiple choice question requires the quiz taker to select the single correct choice from a maximum of four possible choices. A fill in the blank question is the question with a blank field into which the quiz taker must type an answer. The quiz taker will get positive points if he or she types one of the acceptable answers, otherwise negative points will be applied. The answer is not case sensitive, but extra spaces and punctuations are not allowed. A sequence question requires the quiz taker to arrange the items in the required order. There is a maximum of six sequence items. All items must be in the proper sequence for the question. A word bank question enables you to create the question in close style, which asks the quiz taker to choose responses from a pool of choices which correspond to the blanks in the question. This kind of question may look like fill in the blank, and the user need to be careful to avoid ambiguity. A click map question requires the quiz taker to click on the correct areas within an image. Positive Points will be awarded only when all the correct areas are clicked, otherwise negative points will be applied. 
JOURNAL OF VETERINARY MEDICAL RESEARCH 2018, 25 (1): 100-106

\section{Table 1 Specimens and the number of associated landmarks}

\begin{tabular}{|l|l|l||l|l|l|}
\hline No. & \multicolumn{1}{|c|}{ Specimen } & Landmarks & No. & Specimen & Landmarks \\
\hline 1 & $\begin{array}{l}\text { Whole } \\
\text { skeleton }\end{array}$ & 9 & 9 & Carpometacarpus & 11 \\
\hline 2 & $\begin{array}{l}\text { Skull and } \\
\text { Mandible }\end{array}$ & 23 & 10 & Digits of the wing & 11 \\
\hline 3 & Scapula & 4 & 11 & Pelvic bone & 20 \\
\hline 4 & Clavicle & 2 & 12 & Femur & 9 \\
\hline 5 & Coracoid & 6 & 13 & Tibiotarsus and fibula & 17 \\
\hline 6 & Humerus & 11 & 14 & Tarsometatarsus & 5 \\
\hline 7 & Ulna & 7 & 15 & Digits (Toes) & 5 \\
\hline 8 & Radius & 2 & & \\
\hline & & & & \\
\hline
\end{tabular}

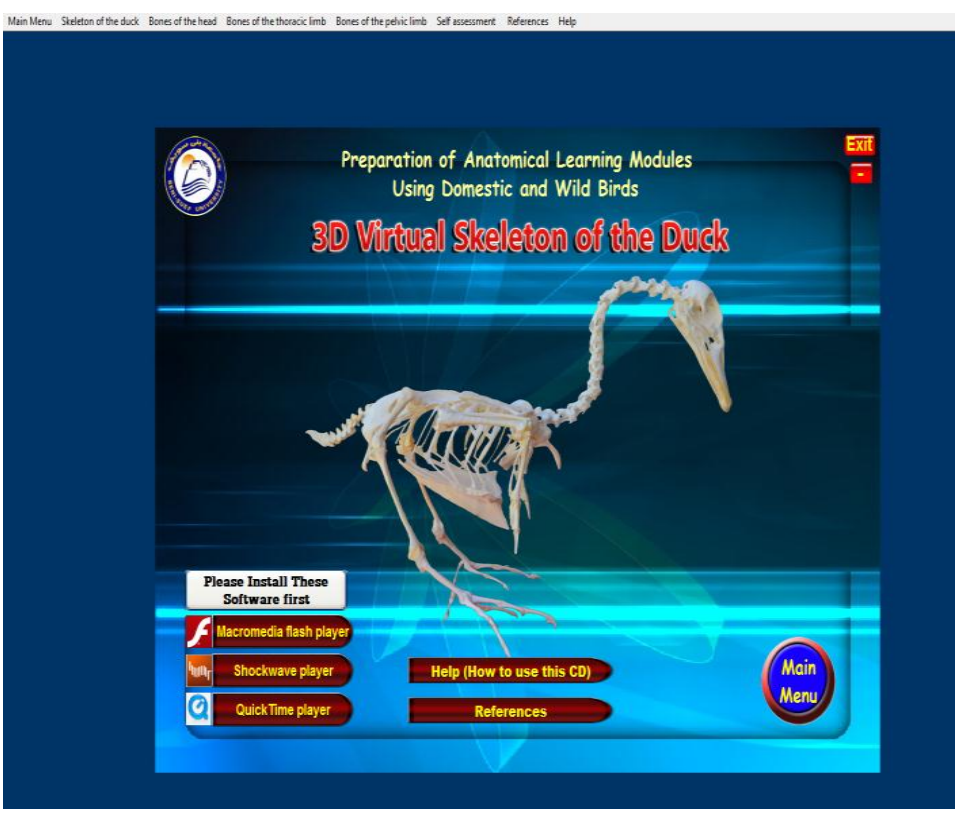

Fig. 1: A printed screen of the introduction page showing the title of the program, the user can access help (How to use this CD), show references used during the preparation of the program, install required software forproper access to the entire program use the dropdown

menus from the menu bar on the top of the screen and navigate the main menu of the program.

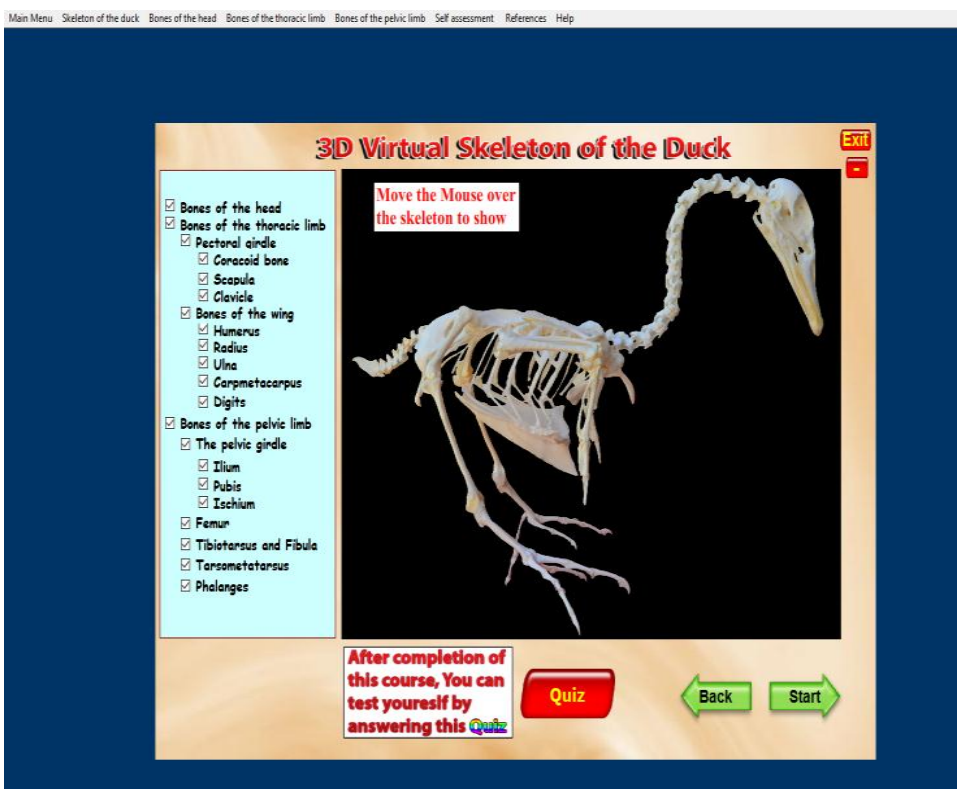

Fig. 2: A printed screen of the main menu of the program with the three buttons: Start, Back, and Quiz. On the left side of the screen, there is a list of the prepared bones from duck skeleton, and on the centre of the screen, there is $2 \mathrm{D}$ image when rollover the mouse on any part, it will be highlighted with different color and information will displayed beside it. 


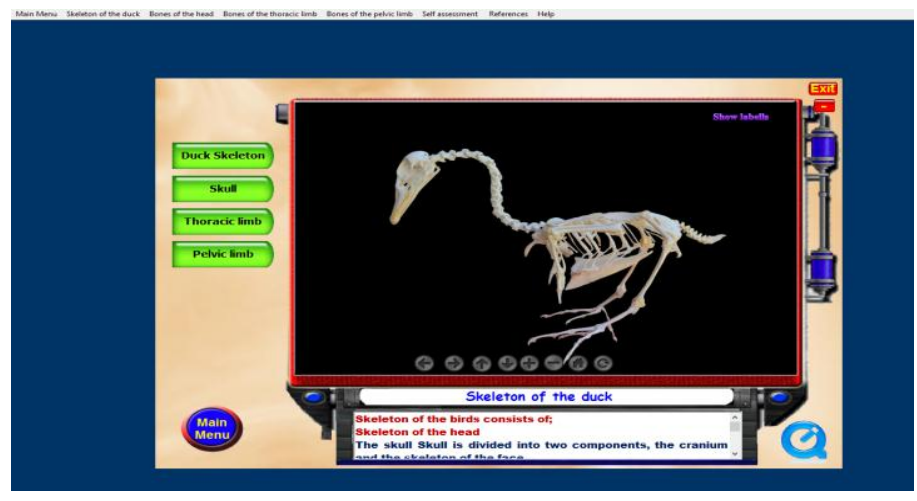

Fig. 3: A printed screen from the main program showing the 3D specimen in the centre, and the eight control buttons below it, (left, right, labels, hide labels, zoom in, zoom out, first view, auto rotate). On the left of the screen, four buttons to navigate different parts of the skeleton, and a multistate button to return back to the main menu.

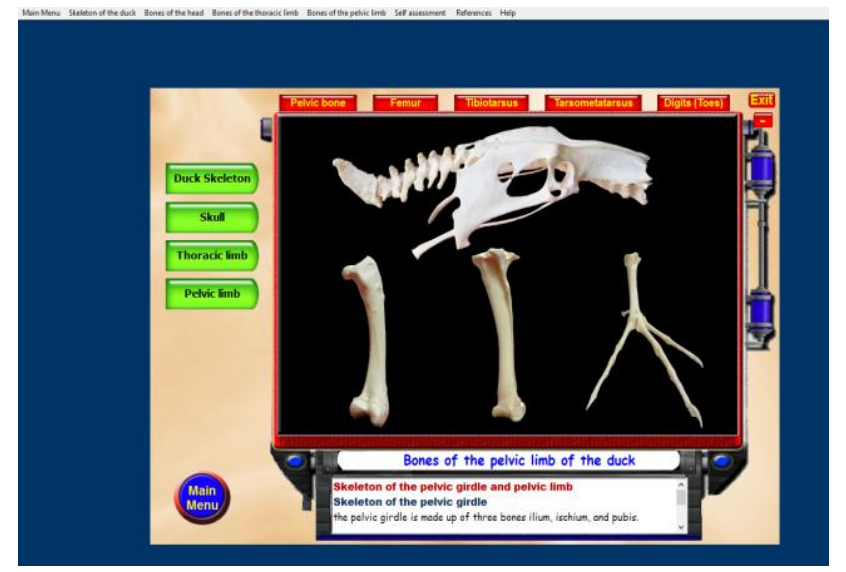

Fig. 5: A printed screen of the program showing the bones of the pelvic limb with the five buttons above the 3D specimen labeled: Pelvic bone, Femur, Tibiotarsus, Tarsometatarsus, and Digits (Toes).

\section{Discussion}

Several attempts were done to minimize the use of animal cadavers in teaching anatomy. This was attributed to the harmful effect of formalin on the instructors, students and workers. Moreover, organizations of animal rights and welfare restrict the animals use from non-ethical sources. Hence, alternative interactive teaching methods such as computer based programs and anatomical modules have been evolved and successfully used El-Nady et al., (2014).

Computer-generated models of the bones are not new, but only very recently has become possible to create bone model that provides realistic, interactive anatomic information with true visualization. This new teaching tool has far-reaching implications not only for students

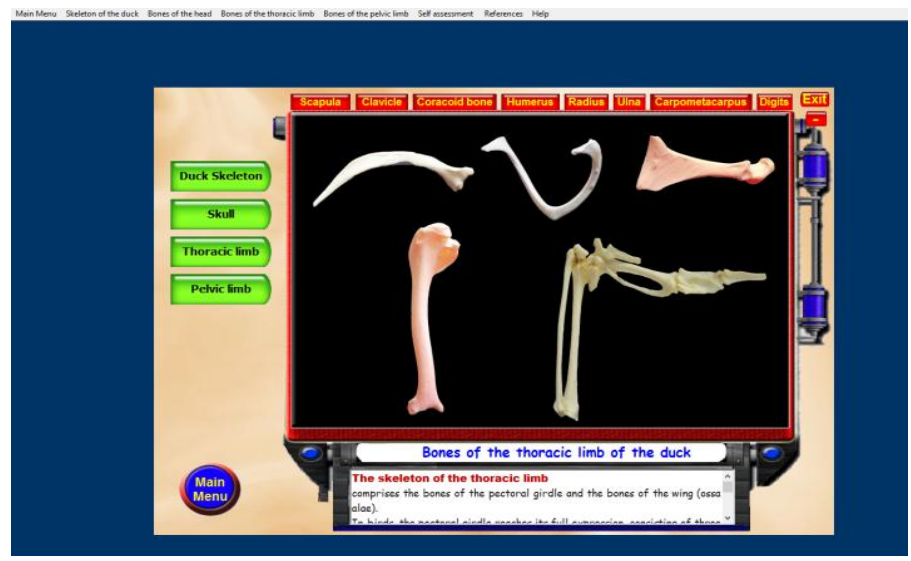

Fig. 4: A printed screen of the program showing the bones of the thoracic limb with eight buttons above the 3D specimen labeled: Scapula, Clavicle, Coracoid bone, Humerus, Radius, Ulna, carpometacarpus and digits.

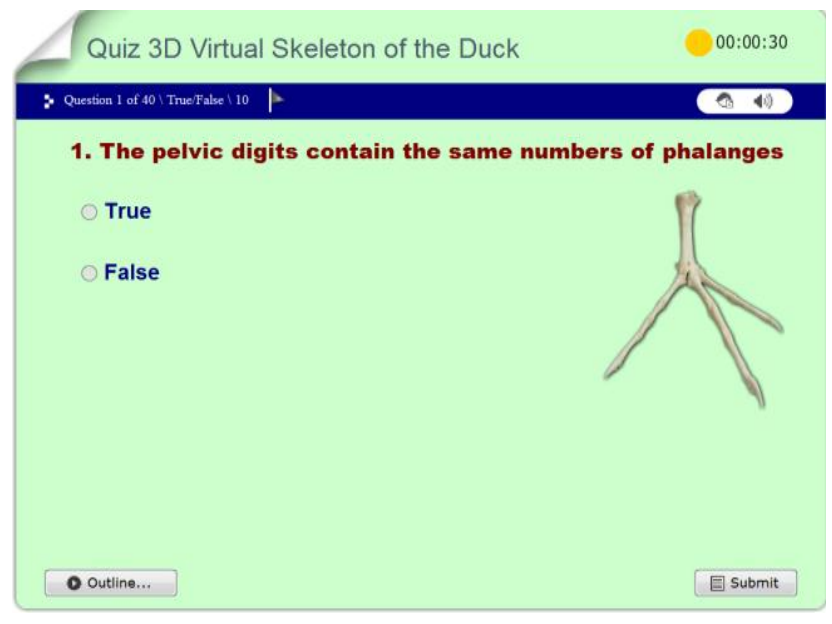

Fig. 6: A printed screen from the quiz window showing "True/false question."

attempting to learn anatomy but also for their teachers and institutions Malinowski (2003), Tawfiek (2011) and ElNady et al., (2014).

Although there will never be a true substitute for the real bones in the anatomy laboratory, we believe that this technology will aid the student to more quickly and easily conceptualize the complexities of bones anatomy. This, in turn, will greatly hasten the student's progression through the laboratory syllabus and allow for a more thorough understanding of anatomic inter relationships.

Technology available today allows for the creation of digital anatomical specimens from a wide variety of samples. Different methods such as photography and radiographic imaging can be used to establish threedimensional models from specimens both living and preserved. In order to produce the virtual specimens in the 
current project, we tried to use all commonly available technologies such as computers, digital cameras

for photography process and software for photo editing and stitching.

These virtual 3D specimens have several advantages over the two-dimensional images and even their physical counterparts. In this connection, Malinowski (2003) mentioned that digital specimens could be distributed very inexpensively using recordable media such as CD-ROM or DVD and can even be incorporated into web pages or downloaded as interactive programs. Three-dimensional models allow the student to interact with the specimens being studied with few simple movements of a computer mouse and the sample can be rotated as if it existed in the student's hand.

The bones in the definitive version of the current work included models of almost every bone of the duck skeleton. This is in strong contrast against other programs such as that of Malinowski (2003) which includes far fewer specimens.

The current project includes more than one type of media such as text, graphics, 3D, flash movies and QuickTime movies and the integration of these entire media is transparent to the user to enhance the effectiveness of the teaching tool. Moreover, the current project provides a high degree of interactivity that makes the user an active participant in the learning process and no longer controlled by the scope of the instructor. This is achieved in the current educational tool, where the learner is in control accessing what he wants, when he wants.

During the construction of the interface of this project, we tried to make it easy for the user to access the information provided with minimum effort. Thus, the interface elements are clearly defined, well organized and permanent through-out the multiple windows of the project.

The use of the prepared 3D virtual specimens from duck skeleton as an educational tool for teaching veterinary anatomy to veterinary students appears to be an effective tool, because it contains complete information about the skeleton. Each bone can easily be rotated and viewed from different angles. Clear labels can be added or removed by the user during display to view the underlying structure with and without the label. A list of the landmarks of each bone is available. A full written text contains all information about the bone. Therefore, the user can find everything he needs in the same screen.

\section{Conclusion}

During the preparation of 3D virtual duck skeleton program, we tried to develop an effective curricular tool that permits reduction in the consumptive use of animals. Because the use of animals in teaching laboratories is currently oriented to transfer established information to a new group of students. The presented sample of alternatives in the current work aimed to replace many of the interventive uses of animals with resources and methods that are equally effective in imparting the essential knowledge and skills about gross anatomy of birds' skeleton.

References

Azer, S.A. and Azer, S. (2016). 3D Anatomy Models and Impact on Learning: A Review of the Quality of the Literature. Health Prof. Educat., 2, 80-98.

Azkue, J.J. (2013). A digital tool for three-dimensional visualization and annotation in anatomy and embryology learning. Eur. J. Anat., 17, 146-154.

Codd, A.M. and Choudhury, B. (2011). Teaching of Human Forearm Musculo-skeletal Anatomy. Anat. Sci. Edu., 4, 119-125.

El-Nady, F.; Rezk, H.M. and Abou Elela, Y.S. (2014). Development of a computer program demonstrating the surface anatomy of the equine' thoracic limb. Vet. Med. J. Giza (VMJG), 60, 51-60.

Fairén, M.; Farrés, M.; Moyés, J. and Insa E. (2017). Virtual Reality to teach anatomy. Eurographics Proc., 51:58.

Galland, J.C.; Oberst, R.D.; Lorenz, M.D. and Mosier, D.A. (1995). Interactive multimedia and casebased learning in Veterinary Medicine- the quantum leap approach. J. Vet. Med. Edu., 22,12-16.

Hildebrand, M. (1968). Anatomical prepara-tions. Univ. California press, Berkley and Los Angles.

König, H.E.; Korbel, R. and Liebich, H.G. (2016). Avian Anatomy, textbook and colour atlas. 5M Publishing Ltd, UK.

Malinowski, R. (2003). 3D K9: Using Quick Time VR to teach veterinary anatomy. Master thesis, Department of Telecomm-unication, Michigan State Univ.

McLelland, J. (1991). A color atlas of avian anatomy. W.B. Saunders Co.

Nickel, R.; Schummer, A. and Seiferle, E. (1977): Anatomy of the domestic birds. Volume 5, Verlag Paul Parey, Springer Verlag.

Tawfiek, M.G. (2011). Preparation of audio-visual aids for use in teaching veterinary anatomy. $\mathrm{PhD}$ thesis, joint between Beni-Suef Univ., Egypt and Virginia Maryland Regional College of Vet. Med., Blacksburg, Virginia Tech., VA., USA.

Turney, B.W. (2007). Anatomy in a modern medical curriculum. Ann. R. Coll. Surg. Engl., 89,104-107. 